\title{
Summary of the NACI Supplemental Statement on Mammalian Cell Culture-Based Influenza Vaccines
}

\author{
Angela Sinilaite ${ }^{1}$, lan Gemmill ${ }^{2,3}$, Robyn Harrison ${ }^{4,5}$ on behalf of the National Advisory Committee \\ on Immunization (NACl)*
}

\section{Abstract}

Background: Mammalian cell culture-based technology is an innovative technique for influenza vaccine manufacturing that may be a valuable alternative to overcome some of the problems and vulnerabilities associated with conventional egg-based influenza vaccine production. Flucelvax ${ }^{\circledast}$ Quad (Seqirus, Inc.) is the first and only mammalian cell culture-based quadrivalent inactivated, subunit influenza vaccine (IIV4-cc) authorized for adult and pediatric use in Canada. The National Advisory Committee on Immunization (NACl) has not previously made a recommendation on cell culture-based influenza vaccines in any population.

Objective: To review the available evidence for the efficacy, effectiveness, immunogenicity, and safety of IIV4-cc, and to summarize the NACI recommendation regarding the use of Flucelvax Quad in Canada in adults and children.

Methods: A systematic literature review on the vaccine efficacy, effectiveness, immunogenicity and safety of IIV4-cc in persons four years of age and older was performed. The systematic review's methodology was specified a priori in a written protocol. The $\mathrm{NACl}$ evidence-based process was used to assess the quality of eligible studies, summarize and analyze the findings, and develop a recommendation regarding the use of Flucelvax Quad in adults and children. The proposed recommendation was then considered and approved by $\mathrm{NACl}$ in light of the available evidence.

Results: Thirteen eligible studies were included in the evidence synthesis. In the four observational studies that assessed vaccine effectiveness of IIV4-cc, there were some data indicating potentially improved protection against influenza compared to conventional egg-based quadrivalent inactivated influenza vaccines (IIV4) or trivalent inactivated influenza vaccine (IIV3), particularly against $A(H 3 N 2)$ virus infection. There was also some evidence that IIV4-cc may be more effective than egg-based trivalent or quadrivalent influenza vaccines against non-laboratory confirmed influenza-related outcomes, but there is insufficient evidence for laboratory-confirmed outcomes. Two randomized controlled trials assessed the immunogenicity and safety of IIV4-cc compared with mammalian cell culture-based trivalent inactivated, subunit influenza vaccine (IIV3-cc). The IIV4-cc was well-tolerated and the reported solicited local and systemic adverse events were generally mild to moderate in intensity, self-limited and did not precipitate sequelae. One clinical review of cases and six peer-reviewed randomized controlled trials (four in adults and two in children) that reported on the safety of IIV3-cc were included in the review. The evidence on immunogenicity and safety was consistent across these studies and showed that there was no significant difference in adults and children four years of age and older who had received IIV3-cc or an egg-based IIV3.

Conclusion: $\mathrm{NACl}$ concluded that there is fair evidence (Grade B Evidence) that Flucelvax Quad is effective, safe, and has non-inferior immunogenicity to comparable vaccines, based on direct evidence in adults and children nine years of age and older. $\mathrm{NACl}$ recommends that Flucelvax Quad may be considered among the IIV4 offered to adults and children nine years of age and older (Discretionary NACI Recommendation).
This work is licensed under a Creative Commons Attribution 4.0 International License.

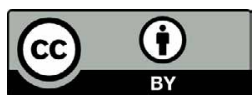

Affiliations

${ }^{1}$ Centre for Immunization and Respiratory Infectious Diseases, Public Health Agency of Canada, Ottawa, ON

${ }^{2} \mathrm{NACl}$ Influenza Working Group Chair

${ }^{3}$ Queen's University, Kingston, $\mathrm{ON}$

${ }^{4} \mathrm{NACl}$ Influenza Working Group Vice Chair

${ }^{5}$ University of Alberta; Alberta Health Services, Edmonton, $A B$

*Correspondence:

phac.naci-ccni.aspc@canada.ca 
Suggested citation: Sinilaite A, Gemmill I, Harrison R, on behalf of the National Advisory Committee on Immunization (NACl). Summary of the NACI Supplemental Statement on Mammalian Cell Culture-Based Influenza Vaccines. Can Commun Dis Rep 2020;46(10):324-32. https://doi.org/10.14745/ccdr.v46i10a03

Keywords: National Advisory Committee on Immunization, NACl, mammalian, cell culture, MDCK, influenza vaccine

\section{Introduction}

Influenza vaccine production using mammalian cell culture-based technology is an innovative technique that may offer enhanced manufacturing scalability, sterility, timeliness and flexibility and, thus, may be a valuable alternative to overcome some of the problems and vulnerabilities associated with conventional egg-based production (1-4). Influenza $A(H 3 N 2)$ viruses can undergo changes that decrease antigenic relatedness to wild-type, circulating viruses when they are grown in eggs, and that certain egg-adaptive mutations may negatively affect the immunogenicity, efficacy and effectiveness of standard egg-based influenza vaccines, especially during influenza $A(H 3 N 2)-d o m i n a n t ~ s e a s o n s ~(4-10)$. Cell culture-based influenza vaccines, solely derived from cell culture-based candidate vaccine viruses (CVVs), are insulated from such egg-adaptive changes and have the potential to provide enhanced protection in some seasons compared to standard egg-based influenza vaccines $(1,4,5)$. Flucelvax Quad (Seqirus, Inc.) is the first and only available mammalian cell culture-based quadrivalent inactivated, subunit influenza vaccine (IIV4-cc) to be authorized for use in Canada in adults and children nine years of age and older (11). Since the vaccine first became available, the Flucelvax quadrivalent formulation (licensed as Flucelvax Quadrivalent or Flucelvax ${ }^{\circledR}$ Tetra in other jurisdictions) has been prepared from viruses propagated in mammalian cell lines (proprietary 33016-PF Madin-Darby Canine Kidney [MDCK] cell lines) adapted to grow freely in suspension in culture medium. However, prior to the 2019-2020 influenza season, some of the CVVs provided to the manufacturer had been originally derived in eggs. The Flucelvax quadrivalent formulation for the 2019-2020 influenza season was the first to be manufactured using CVV for all four influenza viruses that were derived solely from mammalian cell lines from the initial virus isolation through to the full manufacture of the vaccine, making the vaccine egg-free (2).

The National Advisory Committee on Immunization ( $\mathrm{NACl}$ ) has not previously made a recommendation on cell culture-based influenza vaccines in any population. The objective of this advisory committee statement is to review the evidence for efficacy, effectiveness, immunogenicity, and safety that is available for Flucelvax Quad, and to provide provincial and territorial health authorities and health care professionals with guidance on its use in adults and children.

\section{Methods}

A systematic literature review on the vaccine efficacy, effectiveness, immunogenicity and safety of IIV4-cc in persons four years of age and older was performed. Mammalian cell culture-based influenza vaccines have been approved for use by the United States (US) Food and Drug Administration in adults and children four years and older since the 2013-2014 influenza season for the last six years and effectiveness, immunogenicity and safety data is currently available for this age group.

The systematic review's methodology was specified a priori in a written protocol that included review questions, search strategy, inclusion and exclusion criteria and quality assessment. NACl's Influenza Working Group (IWG) reviewed and approved the protocol. A search strategy based on the objective was developed in consultation with a federal Reference Librarian from the Health Library of Health Canada and the Public Health Agency of Canada. Searches were restricted to primary research studies and case reports published in English or French, in the EMBASE, MEDLINE, Scopus, ProQuest Public Health and ClinicalTrials.gov electronic databases from inception until February 12, 2019. Registered clinical trials and grey literature from international public health authorities and National Immunization Technical Advisory Groups were also considered.

Two reviewers independently screened the titles and abstracts and eligible full-text articles.

Studies were included if they met the following criteria:

1. The study population or subpopulation consisted of individuals four years of age and older

2. Study assessed efficacy and effectiveness, immunogenicity, or safety of Flucelvax Quad or safety of Flucelvax

3. Primary research studies from peer-reviewed scientific literature

4. Case reports and case series

5. Registered clinical trials and grey literature from international public health authorities

6. The study was published in English or French

Studies were excluded if they met one or more of the following criteria:

1. The study did not present data on any of: efficacy, effectiveness, immunogenicity or safety of Flucelvax Quad, or the safety of Flucelvax

2. The study is in a language other than English or French

3. The study is a non-human or in vitro study 
4. The article is not a primary research study

5. The article is an editorial, opinion, commentary or news report

6. The article is an economic study, clinical practice guidelines, consensus conference or health technology assessment report

7. The article was a doctoral dissertation, master's thesis or conference summary

Flucelvax Quad has overlapping composition with Flucelvax, which is a trivalent cell culture-based influenza vaccine (IIV3-cc) produced using the same MDCK manufacturing platform $(12,13)$. Therefore, studies that assessed the safety of Flucelvax were also included in this literature review post hoc to supplement the evidence base for the safety outcome. Specialty trivalent vaccines (i.e. high-dose trivalent inactivated influenza vaccine [IIV3-HD] and adjuvanted trivalent inactivated influenza vaccine [IIV3-adj]) were also added as comparator vaccines post hoc, since these comparisons would originally have been excluded as there is currently no comparable quadrivalent formulation of these vaccines.

Data from included studies were extracted into evidence tables using a piloted data abstraction template. The quality (internal validity) of included studies was assessed using criteria outlined by Harris et al. (14). Data extraction and quality assessment were completed by one reviewer and verified by a second reviewer. Results from included studies were synthesized narratively and analyzed according to $\mathrm{NACl}$ evidence-based process (15) to develop a new recommendation. Following thorough review of the evidence, $\mathrm{NACl}$ approved the recommendation.

\section{Results}

The systematic review retrieved 827 unique articles, of which thirteen were retained for data extraction and analysis. Four studies reported on the effectiveness of IIV4-cc. Two peerreviewed studies (one in adults and one in children) investigated the immunogenicity and safety of IIV4-cc. No studies that assessed the immunogenicity of Flucelvax Quad compared to egg-based IIV (trivalent or quadrivalent) were identified. One clinical review of cases and six peer-reviewed randomized controlled trials (RCTs) (four in adults and two in children) provided evidence for the safety of IIV3-cc. No efficacy studies for IIV4-cc were identified and studies evaluating the efficacy of IIV3-cc were beyond the scope of the systematic review. A flow diagram of the study selection process is presented in Figure 1. Key study characteristics are summarized in Tables 1 and 2 .
Figure 1: PRISMA flow diagram of the study selection process for the systematic review on the efficacy, effectiveness, immunogenicity and safety of Flucelvax Quad

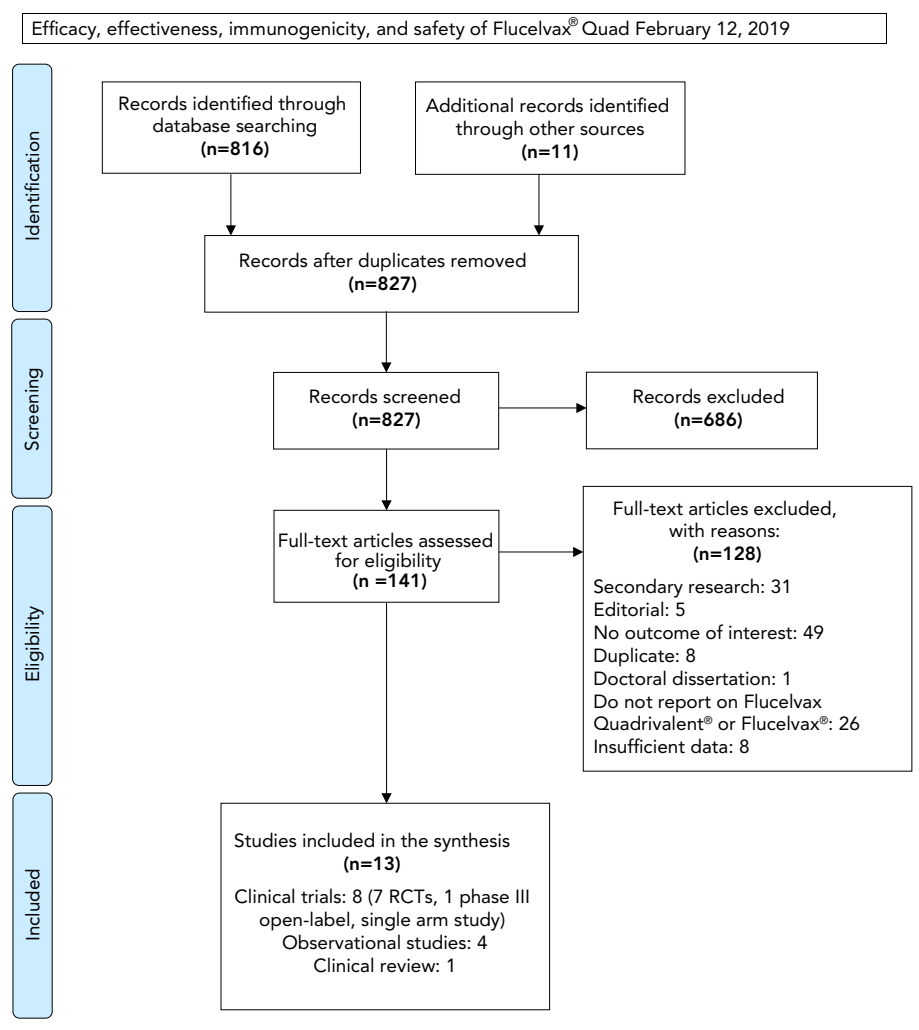

Table 1: Characteristics of IIV4-cc studies included in the systematic review

\begin{tabular}{|c|c|c|c|}
\hline Study & $\begin{array}{l}\text { Design } \\
\text { (vaccine) }\end{array}$ & $\begin{array}{c}\text { Study } \\
\text { population }\end{array}$ & Outcomes \\
\hline $\begin{array}{l}\text { DeMarcus et } \\
\text { al. (2019) }\end{array}$ & $\begin{array}{l}\text { - Test-negative } \\
\text { case-control } \\
\text { - } 2017-2018 \\
\text { influenza } \\
\text { season } \\
\text { - } \text { (IIV4-cc versus } \\
\text { egg-based } \\
\text { IIV4) }\end{array}$ & $\begin{array}{l}\text { - US DOD } \\
\text { healthcare } \\
\text { beneficiaries } \\
\text { six months-94 } \\
\text { years of age } \\
\text { 1,757 cases } \\
\text { (laboratory- } \\
\text { confirmed): } \\
\text { - } 531 \\
\text { vaccinated; } \\
\text { IIV4-cc } \\
\text { ( } n=192) \text {, egg- } \\
\text { based IIV4 } \\
\text { ( } n=339 \text { ) } \\
\text { 2,280 controls: } \\
-977 \\
\text { vaccinated; } \\
\text { IIV4-cc } \\
\text { ( } n=314 \text { ), egg- } \\
\text { based IIV4 } \\
\text { ( } n=663 \text { ) }\end{array}$ & $\begin{array}{l}\text { VE against } \\
\text { laboratory- } \\
\text { confirmed } \\
\text { influenza } \\
\text { - OR for individuals } \\
\text { vaccinated with } \\
\text { cell-derived } \\
\text { vaccine compared } \\
\text { to egg-derived } \\
\text { vaccine }\end{array}$ \\
\hline
\end{tabular}


Table 1: Characteristics of IIV4-cc studies included in the systematic review (continued)

\begin{tabular}{|c|c|c|c|}
\hline Study & $\begin{array}{l}\text { Design } \\
\text { (vaccine) }\end{array}$ & $\begin{array}{c}\text { Study } \\
\text { population }\end{array}$ & Outcomes \\
\hline $\begin{array}{l}\text { Izurieta et al. } \\
(2018)\end{array}$ & $\begin{array}{l}\text { - } \text { Retrospective } \\
\text { cohort } \\
\text { - } 2017-2018 \\
\text { influenza } \\
\text { season } \\
\text { - } \text { (IIV4-cc vs } \\
\text { egg-based } \\
\text { IIV4-SD, } \\
\text { egg-based } \\
\text { IIV3-SD, } \\
\text { IIV3-adj, } \\
\text { IIV3-HD) }\end{array}$ & $\begin{array}{l}\text { Medicare } \\
\text { beneficiaries } \\
65 \text { years of age } \\
\text { and older } \\
\text { - IIV4-cc } \\
\text { ( } n=653,099) \\
\text { - Egg-based } \\
\text { IIV4-SD } \\
\text { ( } n=1,844,745) \\
\text { - Egg-based } \\
\text { IIV3-SD } \\
\text { ( } n=8,449,508) \\
\text { - IIV3-adj: } \\
\text { ( } n=1,465,747) \\
\text { - IIV3-HD } \\
\text { ( } n=1,007,082)\end{array}$ & $\begin{array}{l}\text { - rVE for influenza- } \\
\text { related health } \\
\text { care interactions }\end{array}$ \\
\hline $\begin{array}{l}\text { Boikos et al. } \\
\text { (2018) } \\
\text { NCT } 01992094\end{array}$ & $\begin{array}{l}\text { - } \text { Retrospective } \\
\text { cohort } \\
\text { - } \text { (IIV4-cc vs } \\
\text { egg-based } \\
\text { IIV4) } \\
\text { - } 2017-2018 \\
\text { influenza } \\
\text { season }\end{array}$ & $\begin{array}{l}\text { EMR of US } \\
\text { patients four } \\
\text { years of age or } \\
\text { older } \\
\text { - IIV4-cc } \\
\text { ( } n=92,192) \\
\text { - Egg- } \\
\text { based IIV4 } \\
\text { ( } n=1,255,983)\end{array}$ & $\begin{array}{l}\text { - Propensity-score } \\
\text { matched rVE } \\
\text { against ILI }\end{array}$ \\
\hline $\begin{array}{l}\text { Klein et al. } \\
\text { (2018) }\end{array}$ & $\begin{array}{ll}\text { - } & \text { Retrospective } \\
& \text { cohort } \\
\text { - } & 2017-2018 \\
\text { influenza } \\
\text { season } \\
\text { - } \\
\text { (IIV4-cc vs } \\
\text { egg-based IIV) }\end{array}$ & $\begin{array}{l}\text { Kaiser } \\
\text { Permanente } \\
\text { members 4-64 } \\
\text { years of age } \\
\text { - IIV4-cc } \\
\quad(n=932,874) \\
\text { - Egg-based IIV } \\
(n=84,440)\end{array}$ & $\begin{array}{l}\text { - rVE against } \\
\text { laboratory- } \\
\text { confirmed } \\
\text { influenza A(H3N2) } \\
\text { - rVE against } \\
\text { all laboratory- } \\
\text { confirmed } \\
\text { influenza }\end{array}$ \\
\hline $\begin{array}{l}\text { Bart et al. } \\
\text { (2016) }\end{array}$ & $\begin{array}{ll}\text { - } & \text { RCT } \\
\text { - } & 2013-2014 \\
& \text { influenza } \\
& \text { season } \\
- & \text { (IIV4-cc vs } \\
& \text { IIV3-cc) }\end{array}$ & $\begin{array}{l}\text { Healthy adults } \\
18 \text { years of age } \\
\text { and older } \\
\text { - IIV4-cc } \\
(n=1,335) \\
\text { - IIV3-cc, B/Yam } \\
\text { ( } n=676) \\
\text { - IIV3-cc, B/Vic } \\
\text { ( } n=669)\end{array}$ & $\begin{array}{l}\text { - GMT ratio } 22 \text { days } \\
\text { - Sost-vaccination } \\
\text { - Seroconversion } \\
\text { rate three weeks } \\
\text { (day 22) post- } \\
\text { vaccination } \\
\text { - HI antibody } \\
\text { response } \\
\text { - Seroprotection } \\
\text { rate } \\
\text { - Solicited AEs } \\
\text { within seven days } \\
\text { of vaccination } \\
\text { - Unsolicited AEs } \\
\text { (day 1-22 post- } \\
\text { vaccination) }\end{array}$ \\
\hline $\begin{array}{l}\text { Hartvickson et } \\
\text { al. (2015) } \\
\text { NCT01992107 }\end{array}$ & $\begin{array}{ll}- & \text { RCT } \\
- & 2013-2014 \\
& \text { influenza } \\
& \text { season } \\
- & \text { (IIV4-cc vs } \\
& \text { IIV3-cc) }\end{array}$ & $\begin{array}{l}\text { - Healthy children } \\
\text { 4-18 years of } \\
\text { age; stratified } \\
\text { into two age } \\
\text { cohorts: 4-9 } \\
\text { years of age } \\
\text { and 9-18 years } \\
\text { of age } \\
\text { - IIV4-cc } \\
\text { ( } n=1,159) \\
\text { - IIV3-cc, B/Yam } \\
\text { ( } n=593) \\
\text { - IIV3-cc, B/Vic } \\
\text { ( } n=581)\end{array}$ & $\begin{array}{l}\text { - GMT ratio } 22 \text { days } \\
\text { - Sost-vaccination } \\
\text { - Seroconversion } \\
\text { rate three weeks } \\
\text { (day 22) post- } \\
\text { vaccination with } \\
\text { last dose } \\
\text { - Seroprotection } \\
\text { rate } \\
\text { - Solicited AEs } \\
\text { within seven days } \\
\text { of vaccination } \\
\text { - Unsolicited AEs } \\
\text { (within 22 days of } \\
\text { vaccination) }\end{array}$ \\
\hline
\end{tabular}

Abbreviations: $A E$, adverse event; EMR, Electronic medical record; GMT, geometric mean titre; $\mathrm{HI}$, hemagglutination inhibition; IIV, inactivated influenza vaccine; IIV3, trivalent inactivated influenza vaccine; IIV3-adj, adjuvanted trivalent inactivated influenza vaccine; IIV3-cc, cell-culture based trivalent inactivated influenza vaccine; IIV3-HD, high-dose trivalent inactivated influenza vaccine: IIV3-SD, standard-dose trivalent inactivated influenza vaccine; IIV4, quadrivalent inactivated influenza vaccines; IIV4-cc, cell-culture based quadrivalent inactivated influenza vaccine; IIV4-SD, standard-dose quadrivalent inactivated influenza vaccine; ILI, influenza-like illness; NCT, National clinical trial number; OR, odds ratio; RCT, randomized controlled trial; rVE, relative vaccine effectiveness; US DOD, United States Department of Defense; VE, vaccine effectiveness
Table 2: Characteristics of IIV3-cc studies included in the systematic review

\begin{tabular}{|c|c|c|c|}
\hline Study & $\begin{array}{c}\text { Design } \\
\text { (vaccine) }\end{array}$ & $\begin{array}{c}\text { Study } \\
\text { population }\end{array}$ & Outcomes \\
\hline $\begin{array}{l}\text { Ambrozaitis et } \\
\text { al. (2009) }\end{array}$ & $\begin{array}{ll}- & \text { RCT } \\
- & 2005-2006 \\
& \text { influenza } \\
& \text { season } \\
- & \text { (IIV3-cc } \\
\text { versus egg- } \\
\text { based IIV3) }\end{array}$ & $\begin{array}{l}\text { - Healthy adults } \\
\text { 18-60 years } \\
\text { of age } \\
\text { IIV3-cc } \\
\text { ( } n=1,028) \\
\text { Egg-based } \\
\text { IIV3 }(n=171)\end{array}$ & $\begin{array}{l}\text { - AEs within } \\
\text { seven } \\
\text { days of } \\
\text { vaccination }\end{array}$ \\
\hline $\begin{array}{l}\text { Szymczakiewicz- } \\
\text { Multanowska et } \\
\text { al. (2009) } \\
\text { NCT00492063 }\end{array}$ & $\begin{array}{l}\text { Phase III, } \\
\text { observer } \\
\text { blind RCT } \\
\text { - } 2004-2005 \\
\text { influenza } \\
\text { season } \\
\text { - (IIV3-cc vs } \\
\text { egg-based } \\
\text { IIV3) }\end{array}$ & $\begin{array}{l}\text { - Healthy adults } \\
18 \text { years of } \\
\text { age and older } \\
\text { - IIV3-cc: } \\
\text { - 18-60 years } \\
\text { of age } \\
\text { ( } n=652 \text { ) } \\
\text { - 61 years } \\
\text { of age } \\
\text { and older } \\
\text { ( } n=678 \text { ) } \\
\text { Egg-based } \\
\text { IIV3: } \\
\text { - 18-60 years } \\
\text { of age } \\
\text { ( } n=648 \text { ) } \\
\text { - 61 years } \\
\text { of age } \\
\text { and older } \\
\text { ( } n=676 \text { ) }\end{array}$ & $\begin{array}{l}\text { - AEs within } \\
\text { seven } \\
\text { days of } \\
\text { vaccination }\end{array}$ \\
\hline $\begin{array}{l}\text { Nolan et al. } \\
2016\end{array}$ & $\begin{array}{l}\text { Phase III, } \\
\text { observer } \\
\text { blind RCT } \\
\text { - } 2013-2014 \\
\text { influenza } \\
\text { season } \\
\text { - (IIV3-cc vs } \\
\text { egg-based } \\
\text { IIV3) }\end{array}$ & $\begin{array}{l}\text { - Healthy } \\
\text { children and } \\
\text { adolescents } \\
4-17 \text { years of } \\
\text { age } \\
\text { - IIV3-cc } \\
\text { (n=1,372) } \\
\text { - Egg-based } \\
\text { IIV3 }(n=683)\end{array}$ & $\begin{array}{l}\text { - AEs within } \\
\text { seven } \\
\text { days of } \\
\text { vaccination }\end{array}$ \\
\hline $\begin{array}{l}\text { Vesikari et al. } \\
\text { (2012) }\end{array}$ & $\begin{array}{l}\text { Phase II/III, } \\
\text { observer- } \\
\text { blind RCT } \\
\text { - October } \\
\text { 2007-July } \\
2008 \\
\text { - (IIV3-cc vs } \\
\text { egg-based } \\
\text { IIV3) }\end{array}$ & $\begin{array}{l}\text { - Healthy } \\
\text { children and } \\
\text { adolescents } \\
\text { 3-17 years of } \\
\text { age } \\
\text { - IIV3-cc two } \\
\text { doses 3-8 } \\
\text { years of age } \\
\text { (n=1,599) } \\
\text { - IIV3-cc single } \\
\text { dose 9-17 } \\
\text { years of age } \\
\text { (n=652) } \\
\text { Egg-based } \\
\text { IIV3 3-8 } \\
\text { years of age } \\
\text { ( } n=1,013 \text { ) } \\
\text { Egg-based } \\
\text { IIV3 9-17 } \\
\text { years of age } \\
\text { (n=316) }\end{array}$ & $\begin{array}{l}\text { - AEs within } \\
\text { seven } \\
\text { days of } \\
\text { vaccination }\end{array}$ \\
\hline $\begin{array}{l}\text { Frey et al. } \\
(2010) \\
\text { NCT00630331 }\end{array}$ & $\begin{array}{ll}\text { - Observer- } \\
\text { blind RCT } \\
\text { 2007-2008 } \\
\text { influenza } \\
\text { season } \\
\text { (IIV3-cc vs } \\
\text { egg-based } \\
\text { IIV3) }\end{array}$ & $\begin{array}{l}\text { - Healthy adults } \\
\text { 18-49 years } \\
\text { of age } \\
\text { - } \text { IIV3-cc } \\
\text { ( } n=3,813) \\
\text { - Egg-based } \\
\text { IIV3 ( } n=3,669) \\
\text { - Placebo } \\
(n=3,894)\end{array}$ & $\begin{array}{l}\text { AEs within } \\
\text { seven } \\
\text { days of } \\
\text { vaccination }\end{array}$ \\
\hline
\end{tabular}


Table 2: Characteristics of IIV3-cc studies included in the systematic review (continued)

\begin{tabular}{|c|c|c|c|}
\hline Study & $\begin{array}{l}\text { Design } \\
\text { (vaccine) }\end{array}$ & $\begin{array}{c}\text { Study } \\
\text { population }\end{array}$ & Outcomes \\
\hline $\begin{array}{l}\text { Loebermann et } \\
\text { al. (2019) } \\
\text { NCT01880697 }\end{array}$ & $\begin{array}{l}\text { - Phase III } \\
\text { open-label, } \\
\text { single-arm, } \\
\text { study } \\
\text { - } 2013-2014 \\
\text { influenza } \\
\text { season } \\
\text { - (IIV3-cc) }\end{array}$ & $\begin{array}{l}\text { - Healthy adults } \\
\text { - IIV3-cc: } \\
\text { - 18-60 years } \\
\text { age }(n=63) \\
\text { - } 61 \text { years } \\
\text { age and } \\
\text { older }(n=63)\end{array}$ & $\begin{array}{l}\text { - AEs } \\
\text { following } \\
\text { vaccination }\end{array}$ \\
\hline $\begin{array}{l}\text { Moro et al. } \\
\text { (2015) }\end{array}$ & 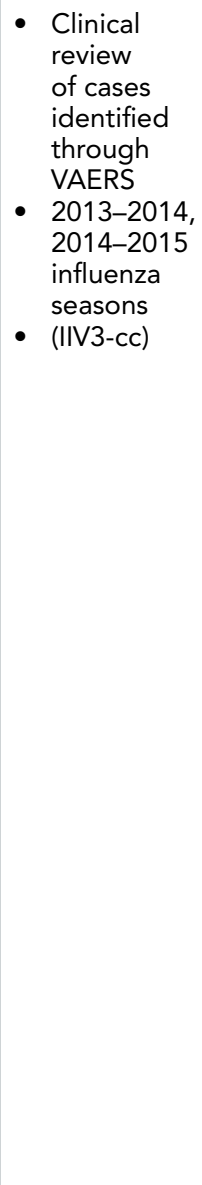 & $\begin{array}{l}\text { - Persons } \\
\text { vaccinated } \\
\text { with IIV3-cc } \\
\text { during July 1, } \\
2013 \text { through } \\
\text { March 31, } \\
2015 \text { (reports } \\
\text { received by } \\
\text { April 30, 2015); } \\
\text { excluding non- } \\
\text { US reports } \\
\text { - Total reports } \\
\text { reviewed: } \\
\text { n=629 } \\
\text { - Persons } \\
\text { vaccinated } \\
\text { with IIV3-cc } \\
\text { July } 1,2013- \\
\text { March } 31, \\
2015 \text { (reports } \\
\text { received by } \\
\text { April 30, 2015); } \\
\text { excluding non- } \\
\text { US reports } \\
\text { - Reports with } \\
\text { an AE: } \\
\text { - n=309 } \\
\text { - during } \\
2013-2014 \\
\text { influenza } \\
\text { season } \\
\text { ( } n=389 \text { ) } \\
\text { - during } \\
2014-2015 \\
\text { influenza } \\
\text { season } \\
\text { ( } n=240 \text { ) }\end{array}$ & $\begin{array}{l}\text { - AEs } \\
\text { following } \\
\text { vaccination }\end{array}$ \\
\hline
\end{tabular}

Abbreviations: $\mathrm{AE}$, adverse event; IIV3, trivalent inactivated influenza vaccine; IIV3-cc, cell-culture based trivalent inactivated influenza vaccine; NCT, National clinical trial number; RCT, randomized controlled trial; US, United States; VAERS, Vaccine Adverse Event Reporting System

\section{Vaccine efficacy and effectiveness}

Four observational studies, two peer-reviewed and two not peer-reviewed, were identified that assessed the vaccine effectiveness of IIV4-cc compared to egg-based IIV against laboratory-confirmed influenza infection during the 2017-2018 influenza season in the US (16-19). Of these four studies, two were of good quality $(17,18)$ according to the criteria outlined by Harris et al. (14), while the quality of the other two studies $(16,19)$ could not be assessed because they were published as conference abstracts or posters. There were some data indicating that IIV4-cc may offer improved protection against influenza compared with conventional egg-based IIV4 or IIV3, particularly against $A(H 3 N 2)$ virus infection. The IIV4-cc may also be more effective than egg-based trivalent or quadrivalent influenza vaccines against non-laboratory confirmed influenza-related outcomes, including influenza-related health care interactions and influenza-like illness, but there was insufficient evidence for laboratory-confirmed outcomes. Although some data suggests that IIV4-cc may be more effective against laboratory-confirmed influenza $A(H 3 N 2)$ virus infection than egg-based IIV, there was no consistent and statistically significant difference in effectiveness identified for adults or children vaccinated with IIV4-cc compared with egg-based IIV.

\section{Immunogenicity}

Two peer-reviewed studies $(20,21)$ that reported on the immunogenicity and safety of Flucelvax Quadrivalent compared with different IIV3-cc formulations were identified in this review; one study by Bart et al. (20) was conducted with adult subjects 18 years of age and older, while the other study by Hartvickson et al. (21) focused on pediatric subjects four to 17 years of age. The immunogenicity outcomes assessed by these studies included geometric mean-fold rise in haemagglutination assay (HA) titres (i.e. ratio of post to pre-vaccination geometric mean titre), seroprotection rate (i.e. proportion of participants with HA titres of at least 40 post-vaccination) and seroconversion rate (i.e. proportion of participants with at least a four-fold increase in HA titres post-vaccination, HA titre increase from less than 10 prevaccination to at least 40 post-vaccination, or both). In both studies, IIV4-cc demonstrated non-inferiority, based on geometric mean titre (GMT) ratio and seroconversion rates, and met the threshold for seroprotection for all influenza strains contained in the IIV3-cc vaccines.

The immunogenicity of Flucelvax Quad is further supported by evidence from the clinical development program for Flucelvax that has been licensed in the US, but for which licensure has never been sought in Canada. Flucelvax has demonstrated non-inferiority to standard egg-based IIV3 comparators for hemagglutination inhibition $(\mathrm{HI})$ antibody responses overall to any strain in adults 18 years of age and older and for $A(H 1 N 1)$ and $B$ strains specifically, but not $A(H 3 N 2)$, for persons four to 17 years of age, based on post-vaccination GMT ratios and seroconversion rates (22-25). Notably, IIV3-cc was manufactured using egg-derived $\mathrm{CV}$ s prior to the implementation of manufacturing methods using $\mathrm{CVV}$ s solely derived from MDCK cells.

\section{Safety}

Two peer-reviewed studies assessed the safety of Flucelvax Quadrivalent (IIV4-cc) compared with different IIV3-cc formulations; one focused on healthy adults (20) and the other on healthy children four years of age and older (21). The safety outcomes assessed included solicited local and systemic adverse events (AE) from day 1-7 post-vaccination, serious adverse events (SAE) through six months after the last vaccination, and unsolicited AEs from day 1-23 post-vaccination. The reported 
solicited local and systemic AE for both adults and children were generally mild to moderate in intensity, self-limited, and did not precipitate sequelae. Serious adverse events were rare and similar in frequency between the quadrivalent and trivalent formulations of the cell culture-based vaccines being compared. No studies that assessed the safety of IIV4-cc compared with egg-based IIV (trivalent or quadrivalent) were identified in this review.

Flucelvax Quadrivalent has been licensed in the US for use in adults and children four years or older in since 2016. Since authorization, no safety signals have been identified through routine pharmacovigilance. One clinical review of cases (26) and six peer-reviewed RCTs $(1,27-31)$ (four in adults and two in children) that reported on the safety of IIV3-cc were included in the review to supplement the evidence base for the safety outcome. The evidence on safety was consistent across studies and showed that there was no significant difference in adults and children four years of age and older who had received the IIV3-cc and egg-based IIV3. Overall, Flucelvax was safe and well tolerated, with local and systemic solicited reactions as well as unsolicited $\mathrm{AE}$ and $\mathrm{SAE}$ comparable to those typically observed with other injectable egg-derived IIV3. The IIV3-cc also has an established record of safety, and no new safety signals have been identified through routine pharmacovigilance in the US or Europe where the vaccine has been licensed $(22,23,25)$.

\section{Discussion}

Flucelvax Quad is considered effective, immunogenic and safe in adults and children nine years of age and older, and has a comparable immunogenicity and safety profile to 1) egg-based influenza vaccines already licensed in Canada and 2) Flucelvax, which is a trivalent cell culture-based influenza vaccine that has been licensed in the US, but for which licensure has never been sought in Canada. The cell culture-based Flucelvax Quad can also provide broader protection against influenza $B$ viruses when compared with trivalent influenza vaccines. There is a theoretical concern that inactivated influenza vaccines produced in canine kidney cells (MDCK 33016-PF) may cause adverse reactions in individuals with dog allergy. However, evidence from in vitro studies on the allergenicity of MDCK cell-based vaccines in individuals with documented allergies associated with dogs, as well as IIV-cc clinical trials and post-market safety surveillance, does not suggest that there is an elevated risk of hypersensitivity reactions as compared with egg-based influenza vaccines $(32,33)$.

Implementation of cell culture-based influenza vaccine technologies and other alternatives to egg-based methods can also enable diversification of vaccine manufacturing platforms to overcome influenza vaccine supply vulnerabilities and improve vaccine-production capacity. Nevertheless, adaptation in cell culture-based influenza vaccines needs to be further investigated given the potential for mutations in the genetic segments of hemagglutinin and neuraminidase surface proteins resulting from serial passaging in MDCK cells $(34,35)$. A more robust, comprehensive and consistent body of evidence, including data on comorbidities, pregnant women, health status, and other potential confounders (36), also needed to evaluate the relative effectiveness and safety of Flucelvax Quad compared with other injectable influenza vaccines. Therefore, ongoing monitoring of vaccine effectiveness, immunogenicity and safety will be important to compare prior and future seasons, across influenza subtypes and overall vaccine effectiveness for each vaccine type.

\section{Limitations}

There are limited peer-reviewed studies currently available on the effectiveness, immunogenicity and safety of IIV4-cc manufactured using fully cell-derived CVVs. All studies that assessed effectiveness were conducted in the US during the same season (2017-2018), which was influenza A(H3N2)dominant. As influenza seasons can vary widely from year to year, interpretation of the data from these observational studies is limited and further evidence on effectiveness gathered during influenza seasons with different circulating viruses is needed before a conclusion on the relative effectiveness can be made. Two of the observational studies $(16,18)$ evaluating vaccine effectiveness utilized real-world primary care data from the electronic medical records of individual patients. The use of electronic medical record datasets for influenza vaccine effectiveness estimation has not yet been validated and the potential sources of bias and confounding still need to be further investigated. Furthermore, the clinical significance and directness of the evidence provided by influenza-related outcomes, which are surrogate measures of influenza activity, remain uncertain.

\section{$\mathrm{NACl}$ recommendation for individual level decision-making}

The following recommendation for Flucelvax Quad supplements $\mathrm{NACl's} \mathrm{overarching} \mathrm{recommendation} \mathrm{for} \mathrm{influenza} \mathrm{vaccination,}$ which is available in the $\mathrm{NACl}$ Seasonal Influenza Vaccine Statement. The overarching $\mathrm{NACl}$ recommendation for influenza vaccination is that an age appropriate influenza vaccine should be offered annually to anyone six months of age and older (Strong NACl Recommendation), noting product-specific contraindications.

1. NACl recommends that Flucelvax Quad may be considered among the IIV4 offered to adults and children nine years of age and older (Discretionary NACI Recommendation)

- $\quad \mathrm{NACl}$ concludes that there is fair evidence to recommend vaccination of adults and children nine years of age and older with Flucelvax Quad (Grade B Evidence) 
The detailed findings of the literature review, and rationale and relevant considerations for this recommendation can be found in the $\mathrm{NACl}$ Supplemental Statement - Mammalian Cell-Culture Based Influenza Vaccines (37).

\section{Conclusion}

There is fair evidence that Flucelvax Quad is effective, safe and has non-inferior immunogenicity to comparable vaccines, based on direct evidence in adults and children nine years of age and older. $\mathrm{NACl}$ recommends that Flucelvax Quad may be considered among the IIV4 offered to adults and children nine years of age and older. $\mathrm{NACl}$ will continue to monitor the evidence related to cell-culture based influenza vaccines and will update this supplemental statement as needed and as data on Flucelvax Quad from several different influenza seasons accumulates.

\section{Authors' statement}

AS - Writing, original draft, review, editing

IG - Review, editing

$\mathrm{RH}-$ Review, editing

The National Advisory Committee on Immunization (NACl) Canadian Immunization Guide Chapter and Statement on Seasonal Influenza Vaccine 2020-2021: Supplemental Statement - Mammalian Cell-Culture Based Influenza Vaccines was prepared by A Sinilaite, J Przepiorkowski, K Young, I Gemmill, and R Harrison on behalf of the NACl Influenza Working Group and was approved by $\mathrm{NACl}$.

\section{Competing interests}

None.

\section{Acknowledgements}

Influenza Working Group members: I Gemmill (Chair), R Harrison (Vice-Chair), C Bancej, L Cochrane, N Dayneka, L Grohskopf, K Klein, D Kumar, J Langley, P Wolfe-Roberge, J McElhaney, A McGeer, D Moore, S Smith, B Warshawsky and J Xiong

NACl members: C Quach (Chair), S Deeks (Vice-Chair), N Dayneka, P De Wals, V Dubey, R Harrison, K Hildebrand, K Klein, J Papenburg, C Rotstein, B Sander and S Smith

Former NACI members: M Salvadori and N Sicard

Liaison representatives: LM Bucci (Canadian Public Health Association), E Castillo (Society of Obstetricians and Gynaecologists of Canada), A Cohn (Centers for Disease Control and Prevention, United States), J Emili (College of Family Physicians of Canada), M Naus (Canadian Immunization Committee), D Moore (Canadian Paediatric Society) and
A Pham-Huy (Association of Medical Microbiology and Infectious Disease Canada)

Ex-officio representatives: J Gallivan (Marketed Health Products Directorate, Health Canada [HC]), E Henry (Centre for Immunization and Respiratory Infectious Diseases [CIRID], Public Health Agency of Canada [PHAC]), M Lacroix (Public Health Ethics Consultative Group, PHAC), J Pennock (CIRID, PHAC), R Pless (Biologics and Genetic Therapies Directorate, $\mathrm{HC}$ ), G Poliquin (National Microbiology Laboratory, PHAC) and T Wong (First Nations and Inuit Health Branch, Indigenous Services Canada)

The National Advisory Committee on Immunization acknowledges and appreciates the contribution of A House (CIRID, PHAC), M Laplante (CIRID, PHAC), and K Merucci (Health Library, $\mathrm{HC}$ ) to this statement.

\section{Funding}

The work of the National Advisory Committee on Immunization is supported by the Public Health Agency of Canada.

\section{References}

1. Frey S, Vesikari T, Szymczakiewicz-Multanowska A, Lattanzi M, Izu A, Groth N, Holmes S. Clinical efficacy of cell culture-derived and egg-derived inactivated subunit influenza vaccines in healthy adults. Clin Infect Dis 2010 Nov;51(9):997-1004. DOl PubMed

2. Centers for Disease Control and Prevention) (US). Seasonal Influenza (Flu): Cell-Based Flu Vaccines. CDC; 2016 (accessed 2019-07-15). https://www.cdc.gov/flu/prevent/cell-based. htm

3. Gregersen JP, Schmitt HJ, Trusheim H, Bröker M. Safety of MDCK cell culture-based influenza vaccines. Future Microbiol 2011;6(2):143-52. DOI PubMed

4. Barr IG, Donis RO, Katz JM, McCauley JW, Odagiri T, Trusheim H, Tsai TF, Wentworth DE. Cell culture-derived influenza vaccines in the severe 2017-2018 epidemic season: a step towards improved influenza vaccine effectiveness. NPJ Vaccines 2018;(3):44 1-5. DOI PubMed

5. Centers for Disease Control and Prevention (US). Department of Health and Human Services Centers for Disease Control and prevention: Advisory Committee on Immunization Practices (ACIP): Summary Report June 20-21, 2018, Atlanta Georgia. ACIP; 2018 (accessed 2019-07-15). https://www.cdc.gov/vaccines/acip/meetings/downloads/ min-archive/min-2018-06-508.pdf

6. Skowronski DM, Chambers C, De Serres G, Dickinson JA, Winter AL, Hickman R, Chan T, Jassem AN, Drews SJ, Charest H, Gubbay JB, Bastien N, Li Y, Krajden M. Early season co-circulation of influenza $\mathrm{A}(\mathrm{H} 3 \mathrm{~N} 2)$ and $\mathrm{B}$ (Yamagata): interim estimates of 2017/18 vaccine effectiveness, Canada, January 2018. Euro Surveill 2018;23(5):pii=18-00035. DOI PubMed 
7. Skowronski DM, Janjua NZ, De Serres G, Sabaiduc S, Eshaghi A, Dickinson JA, Fonseca K, Winter AL, Gubbay JB, Krajden M, Petric M, Charest H, Bastien N, Kwindt TL, Mahmud SM, Van Caeseele P, Li Y. Low 2012-13 influenza vaccine effectiveness associated with mutation in the egg-adapted H3N2 vaccine strain not antigenic drift in circulating viruses. PLoS One 2014 Mar;9(3):e92153. DOI PubMed

8. Zost SJ, Parkhouse K, Gumina ME, Kim K, Diaz Perez S, Wilson PC, Treanor JJ, Sant AJ, Cobey S, Hensley SE. Contemporary $\mathrm{H} 3 \mathrm{~N} 2$ influenza viruses have a glycosylation site that alters binding of antibodies elicited by egg-adapted vaccine strains. Proc Natl Acad Sci USA 2017;114(47):1257883. DOI PubMed

9. Wu NC, Zost SJ, Thompson AJ, Oyen D, Nycholat CM, McBride R, Paulson JC, Hensley SE, Wilson IA. A structural explanation for the low effectiveness of the seasonal influenza H3N2 vaccine. PLoS Pathog 2017;13(10):e1006682. DOI PubMed

10. The Francis Crick Institute. Worldwide Influenza Centre: Annual and Interim Reports - February 2018 interim report. FCl; 2018 (accessed 2019-07-15). https:// www.crick.ac.uk/research/worldwide-influenza-centre/ annual-and-interim-reports/

11. Seqirus UK. Limited. Product monograph: FLUCELVAX ${ }^{\circledR}$ QUAD: Influenza Vaccine (surface antigen, inactivated, prepared in cell cultures). 2019 (accessed 2019-07-15). https://pdf.hres.ca/dpd_pm/00054016.PDF

12. Seqirus. Flucelvax Doctor Discussion Guide. Holly Springs (NC): Seqirus; 2017 (accessed 2019-07-15). https://labeling. cslbehring.com/PRODUCT-DOCUMENT/US/Flucelvax/EN/ Flucelvax-Doctor-Discussion-Guide.pdf

13. Seqirus. US Product Monograph: Flucelvax Quadrivalent. [Internet]. Holly Springs (NC): Seqirus; 2016 (accessed 201907-15). http://labeling.seqirus.com/PI/US/Flucelvax/EN/ Flucelax-Prescribing-Information.pdf

14. Harris RP, Helfand M, Woolf $\mathrm{SH}$, Lohr KN, Mulrow CD, Teutsch SM, Atkins D; Methods Work Group, Third US Preventive Services Task Force. Current methods of the US Preventive Services Task Force: a review of the process. Am J Prev Med 2001;20(3 Suppl):21-35. DOI PubMed

15. National Advisory Committee on Immunization (NACl). Evidence-based recommendations for immunization--methods of the National Advisory Committee on Immunization. An Advisory Committee Statement (ACS). Can Commun Dis Rep. 2009;35(ACS1):1-10. https://www.canada.ca/en/public-health/services/ reports-publications/canada-communicable-disease-reportccdr/monthly-issue/2009-35/methods-nationa l-advisory-committee-immunization.html

16. Boikos C, Sylvester G, Sampalis J, Mansi J. Effectiveness of the Cell Culture-and Egg-Derived, Seasonal Influenza Vaccine during the 2017-2018 Northern Hemisphere Influenza Season. Canadian Immunization Conference 2018; 2018 Dec 4-6; Ottawa, ON, Canada. [Poster presentation]. https://www.izsummitpartners.org/content/uploads/201 9/05/1-effectiveness-of-cell-culture-and-egg-derived-fluvax-during-2017-2018-flu-season.pdf
17. DeMarcus L, Shoubaki L, Federinko S. Comparing influenza vaccine effectiveness between cell-derived and egg-derived vaccines, 2017-2018 influenza season. Vaccine 2019;37(30):4015-21. DOI PubMed

18. Izurieta HS, Chillarige $Y$, Kelman J, Wei Y, Lu Y, Xu W, Lu M, Pratt D, Chu S, Wernecke M MaCurdy T, Forshee R. Relative effectiveness of cell-cultured and egg-based influenza vaccines among elderly persons in the United Sates, 2017-18. J Infect Dis 2019;220(8):1255-64. DOI PubMed

19. Klein NP, Fireman B, Goddard K, Zerbo O, Asher J, Zhou J, King J, Lewis N. Vaccine Effectiveness of Flucelvax Relative to Inactivated Influenza Vaccine During the 2017-18 Influenza Season in Northern California (presentation LB15 at IDSA conference). Open Forum Infect Dis 2018;5 Suppl 1:S764. DOI

20. Bart S, Cannon K, Herrington D, Mills R, Forleo-Neto E, Lindert K, Abdul Mateen A. Immunogenicity and safety of a cell culture-based quadrivalent influenza vaccine in adults: A Phase III, double-blind, multicenter, randomized, non-inferiority study. Hum Vaccin Immunother 2016;12(9):2278-88. DOl PubMed

21. Hartvickson R, Cruz M, Ervin J, Brandon D, Forleo-Neto E, Dagnew AF, Chandra R, Lindert K, Mateen AA. Non-inferiority of mammalian cell-derived quadrivalent subunit influenza virus vaccines compared to trivalent subunit influenza virus vaccines in healthy children: a phase III randomized, multicenter, double-blind clinical trial. Int J Infect Dis 2015;41:65-72. DOI PubMed

22. European Medicines Agency. Assessment report for paediatric studies submitted according to Article 46 of the Regulation (EC) No 1901/2006. Optaflu. EUEMA; 2015 (accessed 2019-07-15). https://www.ema.europa. eu/en/documents/variation-report/optaflu-h-c-758-p460052-epar-assessment-report_en.pdf

23. European Medicines Agency. Optaflu: European Public Assessment Report: Scientific Discussion. EUEMA; 2007 (accessed 2019-07-15). https://www.ema.europa. eu/en/documents/scientific-discussion/optaflu-epa r-scientific-discussion_en.pdf

24. US Food and Drug Administration. Flucelvax Quadrivalent. FDA (updated 2020; accessed 2019-07-15). https://www.fda.gov/vaccines-blood-biologics/vaccines/ flucelvax-quadrivalent

25. US Food and Drug Administration. FLUCELVAX - Seqirus, Inc. 1.14.1.3 US Package Insert. FDA; 2016 (accessed 2019-07-15). https://www.fda.gov/media/85322/download

26. Moro PL, Winiecki S, Lewis $\mathrm{P}$, Shimabukuro TT, Cano M. Surveillance of adverse events after the first trivalent inactivated influenza vaccine produced in mammalian cell culture (Flucelvax ${ }^{\circledR}$ ) reported to the Vaccine Adverse Event Reporting System (VAERS), United States, 2013-2015. Vaccine 2015;33(48):6684-8. DOI PubMed 
27. Vesikari T, Block SL, Guerra F, Lattanzi M, Holmes S, Izu A, Gaitatzis N, Hilbert AK, Groth N. Immunogenicity, safety and reactogenicity of a mammalian cell-culture-derived influenza vaccine in healthy children and adolescents three to seventeen years of age. Pediatr Infect Dis J 2012;31(5):494-500. DOl PubMed

28. Ambrozaitis A, Groth N, Bugarini R, Sparacio V, Podda A, Lattanzi M. A novel mammalian cell-culture technique for consistent production of a well-tolerated and immunogenic trivalent subunit influenza vaccine. Vaccine 2009;27(43):6022-9. DOl PubMed

29. Szymczakiewicz-Multanowska A, Groth N, Bugarini R, Lattanzi M, Casula D, Hilbert A, Tsai T, Podda A. Safety and immunogenicity of a novel influenza subunit vaccine produced in mammalian cell culture. $J$ Infect Dis 2009;200(6):841-8. DOI PubMed

30. Loebermann M, Fritzsche $C$, Geerdes-Fenge $H$, Heijnen $E_{\text {, }}$ Kirby D, Reisinger EC. A phase III, open-label, single-arm, study to evaluate the safety and immunogenicity of a trivalent, surface antigen inactivated subunit influenza virus vaccine produced in mammalian cell culture (Optaflu $\left.{ }^{\circledR}\right)$ in healthy adults. Infection 2019;47(1):105-9. DOI PubMed

31. Nolan T, Chotpitayasunondh T, Capeding MR, Carson S, Senders SD, Jaehnig P, de Rooij R, Chandra R. Safety and tolerability of a cell culture derived trivalent subunit inactivated influenza vaccine administered to healthy children and adolescents: A Phase III, randomized, multicenter, observer-blind study. Vaccine 2016;34(2):230-6. DOI PubMed

32. Bencharitiwong R, Leonard S, Tsai T, Nowak-Węgrzyn A. In vitro assessment of the allergenicity of novel MF59-adjuvanted pandemic H1N1 influenza vaccine produced in dog kidney cells. Hum Vaccin Immunother 2012;8(7):863-5. DOI PubMed
33. Wanich N, Bencharitiwong R, Tsai T, Nowak-Wegrzyn A. In vitro assessment of the allergenicity of a novel influenza vaccine produced in dog kidney cells in individuals with dog allergy. Ann Allergy Asthma Immunol 2010;104(5):426-33. DOI PubMed

34. Harding AT, Heaton NS. Efforts to improve the seasonal influenza vaccine. Vaccines (Basel) 2018 Mar;6(2):19. DOI PubMed

35. Lin Y, Wharton SA, Whittaker L, Dai M, Ermetal B, Lo J, Pontoriero A, Baumeister E, Daniels RS, McCauley JW. The characteristics and antigenic properties of recently emerged subclade $3 \mathrm{C} .3 \mathrm{a}$ and $3 \mathrm{C} .2 \mathrm{a}$ human influenza $\mathrm{A}(\mathrm{H} 3 \mathrm{~N} 2)$ viruses passaged in MDCK cells. Influenza Other Respir Viruses 2017;11(3):263-74. DOI PubMed

36. Remschmidt $\mathrm{C}$, Wichmann $\mathrm{O}$, Harder T. Frequency and impact of confounding by indication and healthy vaccinee bias in observational studies assessing influenza vaccine effectiveness: a systematic review. BMC Infect Dis 2015 Oct;15(429):429. DOI PubMed

37. National Advisory Committee on Immunization (NACl). An Advisory Committee Statement (ACS. Canadian Immunization Guide Chapter and Statement on Seasonal Influenza Vaccine 2020-2021: Supplemental Statement Mammalian Cell-Culture Based Influenza Vaccines. Ottawa (ON): PHAC; 2020. https://www.canada.ca/en/public-health/ services/immunization/national-advisory-committee-o n-immunization-naci/mammalian-cell-culture-base d-influenza-vaccines.html 